\title{
Implications of the IQ-CSRC Prospective Study: Time to Revise ICH E14
}

\author{
Borje Darpo $^{1,2} \cdot$ Christine Garnett $^{3} \cdot$ James Keirns $^{4} \cdot$ Norman Stockbridge $^{3}$
}

Published online: 11 July 2015

(C) Springer International Publishing Switzerland 2015

\begin{abstract}
Exposure-response (ER) analysis has evolved as an important tool to evaluate the effect of a drug on cardiac repolarization, as reflected in the QTc interval. It has been suggested that careful electrocardiogram (ECG) evaluation in 'first-in-human' studies using ER analysis could replace or serve as an alternative to the E14 'thorough QT' study. This commentary shares and discusses the results of a recently conducted study with the objective to evaluate this approach. Six drugs with a well-characterized QT effect, five of which are known QT prolongers, were evaluated in a study design similar to a conventional single-ascending-dose study. Each drug was given to nine healthy subjects (six for placebo) in two dose levels, which for the positive drugs (ondansetron, quinine, dolasetron, moxifloxacin, and dofetilide) were chosen with the intent to cause 10-12 ms and 15-20 ms QTc prolongation. Replicate 12-lead ECGs were extracted from continuous recordings pre-dose and serially after dosing and paired with drug concentration determinations. The ER criteria for
\end{abstract}

This article reflects the views of the authors and should not be construed as representing the views or policies of the US FDA.

\footnotetext{
Borje Darpo

borje.darpo@telia.com

1 Division of Cardiovascular Medicine, Department of Clinical Sciences, Danderyd's Hospital, Karolinska Institutet, Stockholm, Sweden

2 iCardiac Technologies, Inc., Rochester, NY, USA

3 Division of Cardiovascular and Renal Products, Center for Drug Evaluation and Research, US Food and Drug Administration, Silver Spring, MD, USA

4 Global Clinical Pharmacology and Exploratory Development, Astellas Pharma Global Development, Inc., Northbrook, IL, USA
}

the identification of a QT effect, a statistically significant positive ER slope and an effect above $10 \mathrm{~ms}$, were met with all five positive drugs, and an effect exceeding $10 \mathrm{~ms}$ could be excluded at the supratherapeutic dose of the negative drug, levocetirizine. The study results thereby provided evidence to support that careful QT assessment in early phase clinical studies can be used as an alternative to the thorough QT study. Clinical and regulatory implications, as well as limitations of this approach, are discussed in the commentary.

\section{Key Points}

Evaluation of a drug's potential effect on the QT interval can be generated with high confidence using exposure-response analysis of electrocardiogram and pharmacokinetic data from first-in-human studies.

This approach, 'Early QT assessment', can provide an alternative to the thorough QT studies in select cases.

\section{Introduction}

The thorough QT (TQT) study has been a key component of the clinical evaluation of the propensity of new drugs to cause QTc prolongation since the adoption of the International Conference of Harmonisation (ICH) E14 clinical guidance document in May 2005 [1]. The request to study 
each new drug in a specifically designed study in healthy subjects if justifiable from a tolerability and safety perspective, and otherwise in the target patient population, was triggered by a number of drug withdrawals in the 1990s for arrhythmias associated with QT prolongation [2, 3]. The TQT study has been successful in terms of detecting drugs with a QT effect and thereby avoiding the introduction of new drugs with an unknown QT liability to the market. However, this has had its price; based on a conservatively chosen threshold $(10 \mathrm{~ms})$ and the requirement that the QT effect is evaluated separately at each post-dosing timepoint, without consideration of the pharmacology of the drug, the study is overly sensitive and has therefore resulted in a number of 'false' positives, i.e., drugs are labeled as QT prolongers without a demonstrated underlying proarrhythmic risk $[4,5]$. The TQT study is also resource intensive [6], and, if electrocardiogram (ECG) data could be generated with the same level of confidence from other studies routinely performed as part of clinical development, this would represent a more efficient approach, with other potential advantages, such as improved understanding of any liabilities early in clinical development. The 'first-in-human' studies [single ascending dose (SAD) and multiple ascending dose (MAD)] seem well suited for this purpose because achieved plasma levels of the parent compound and abundant metabolites often substantially exceed therapeutic levels later observed in patients. Provided serial ECG assessment and pharmacokinetic sampling are incorporated into the design, SAD and MAD studies represent an opportunity to generate ECG data with the same high quality as the TQT study [79]. Several doses of the investigational drug are typically administered to small cohorts with only six to eight subjects receiving active drug (and often only two per cohort receiving placebo), and the power to exclude small effects in a 'by timepoint' analysis for each dose group as in the TQT study is therefore unacceptably low [10]. If, on the other hand, exposure-response (ER) analysis is employed, all data across a wide range of plasma concentrations of the drug are used, and the power to detect and exclude small QT effects would be substantially improved [11].

The experience with ER analysis of ECG data has increased over the last decade, among both regulators and sponsors. The US FDA Interdisciplinary Review Team (IRT) for QT studies was formed shortly after the adoption of the ICH E14 document and has since provided sponsors with consistent advice on the design and analysis of TQT studies [12] and has independently reviewed and analyzed close to $400 \mathrm{TQT}$ studies to date. ER analysis has become an integral part of the IRT review of data from QT assessment studies $[11,12]$ and has proven invaluable in terms of enhancing the confidence in characterizing druginduced QTc prolongation. ER analysis is now routinely used to predict the QT effect in the targeted patient population, including clinical scenarios with doses and formulations not directly evaluated in the TQT study and QT effects in specific populations and under certain conditions (e.g., drug interactions) with increased exposure of the drug [13-19]. Extensive experience with QT-prolonging drugs demonstrate that the effect on the QT interval is directly related to plasma levels of the drug or main metabolites, with few exceptions (e.g., QT prolongation inhibition of hERG protein trafficking, which is delayed in relation to peak plasma levels [20-22]). In our view, it therefore makes sense to focus on QT effects in relation to plasma concentration of the drug, rather than by timepoint without consideration of the pharmacology of the drug, and a wider role for ER analysis in the assessment of drug-induced ECG effects seems justified.

Even though the experience of many pharmaceutical sponsors from the application of ER analysis on data from first-in-human studies is favorable, publicly available reports are relatively scarce [7-9, 23]. Based on discussions with the FDA, a research collaboration was therefore initiated between the Clinical Pharmacology Leadership Group of the Consortium for Innovation and Quality in Pharmaceutical Development (IQ Consortium) [24] and the Cardiac Safety Research Consortium (CSRC [25]) with the intention of conducting a prospective study to evaluate whether ER analysis applied to ECG data from a small SAD-like clinical pharmacology study could serve as an alternative to the TQT study. In this commentary, an outline of the main results of the study is given and potential implications thereof are discussed.

\section{The IQ-CSRC Prospective Study}

The objective of the IQ-CSRC study was to evaluate whether QT assessment performed in early phase clinical studies using an intense ECG schedule and ER analysis can detect, and therefore also exclude, small QT effects with the same level of confidence as would a TQT study. The selection of drugs, doses, design of the study, and methods of analyses [26] were discussed and agreed upon with the FDA, and the results have recently been published [27]. Healthy subjects $(n=20)$ were enrolled into the study and underwent three separate treatment periods, during which study treatment or placebo was administered on 2 consecutive days. Six drugs with well-characterized QT effect were selected for the evaluation, as follows. Five 'QTpositive' drugs were administered at doses intended to cause QTc prolongation of around 9-12 ms on day 1 and 15-20 ms on day 2: (1) oral ondansetron $52 \mathrm{mg}$ and intravenous ondansetron $32 \mathrm{mg}$ [28]; (2) quinine $648 \mathrm{mg}$ three times daily for four doses [29, 30]; (3) oral dolasetron 
$100 \mathrm{mg}$ and intravenous dolasetron $150 \mathrm{mg}$ [31]; (4) oral moxifloxacin $400 \mathrm{mg}$ and intravenous moxifloxacin $800 \mathrm{mg}[32,33]$; and (5) oral dofetilide 0.125 and $0.25 \mathrm{mg}$ [34]. One negative drug, levocetirizine, was added at the same doses as in its TQT study, 5 and $30 \mathrm{mg}[35,36]$. An incomplete block design resulted in each study drug being administered to nine subjects and placebo to six subjects in separate periods. Serial ECGs and pharmacokinetic samples were collected on each dosing day. The primary variable for the ER analysis was the change from pre-dose baseline QTcF $(\Delta \mathrm{QT} c \mathrm{~F})$. Prospective criteria were used to exclude hysteresis and to select the appropriate ER model. To claim that the study was able to demonstrate the QT effect of the five QT-positive drugs, the slope of the concentration-QTc effect relationship had to be statistically significantly different from zero, and the upper bound of the two-sided $90 \%$ confidence interval (CI) of the predicted mean $\Delta \Delta \mathrm{QTCF}$ had to be greater than $10 \mathrm{~ms}$ at the observed geometric mean peak plasma drug concentration $\left(C_{\max }\right)$ of the lower dose on day 1 . To exclude a QT effect for the 'QT negative' drug (levocetirizine), the upper bound of the two-sided $90 \% \mathrm{CI}$ of the predicted mean $\Delta \Delta \mathrm{QTcF}$ had to be less than $10 \mathrm{~ms}$ at the observed geometric mean $C_{\max }$ after the higher dose (30 mg) on day 2 .

Data were available from eight to nine subjects receiving active drugs on day 1 , from six to nine subjects on day 2 , and from six subjects receiving placebo from both study days. Across all timepoints on day 1, the largest mean placebo-adjusted $\Delta \mathrm{QTcF}(\Delta \Delta \mathrm{QTcF})$ was between 10 and $15 \mathrm{~ms}$ for all QT-positive drugs except hydrodolasetron (the active metabolite of dolasetron) with an effect of $6.5 \mathrm{~ms} ; \Delta \Delta \mathrm{QTcF}$ was $1.8 \mathrm{~ms}$ for levocetirizine. On day 2 , the largest mean $\Delta \Delta \mathrm{QTcF}$ reached 10.2 and $12.2 \mathrm{~ms}$ for ondansetron and hydrodolasetron, respectively, and was above $20 \mathrm{~ms}$ for quinine (22.1 ms), moxifloxacin (33.4 ms), and dofetilide $(24.5 \mathrm{~ms})$; peak $\Delta \Delta \mathrm{QTcF}$ on levocetirizine was $3.1 \mathrm{~ms}$. A linear ER model provided the best fit of the data for all dugs except dofetilide, for which a maximum response $\left(E_{\max }\right)$ model was better according to pre-specified model-selection criteria. A significant slope of the ER relationship was demonstrated, and the upper bound of the $90 \% \mathrm{CI}$ of the predicted effect at the observed $C_{\max }$ of day 1 was above $10 \mathrm{~ms}$ for all positive drugs, i.e., all QT-positive drugs met the prespecified criteria (Table 1; Fig. 1). For the negative drug, levocetirizine, an effect exceeding $10 \mathrm{~ms}$ could be excluded at the observed mean $C_{\max }(1005 \mathrm{ng} / \mathrm{mL})$ of the higher dose, $30 \mathrm{mg}$. Two sensitivity analyses were performed. The first was performed to explore the scenario in which the peak QT effect was at the level of regulatory concern, i.e., the intended effect level on day 1 (9-12 ms). For this purpose, only data from day 1 with the lower doses of the positive drugs and from day 2 with the higher dose of the negative drug were used; criteria for positive and negative QT assessment were still met for all drugs (Table 2; Fig. 2). Since many SAD studies are of pure parallel-group design, active treatment periods for subjects who also received placebo were excluded in the second analysis, in effect, creating a pure parallel-group comparison with six to seven subjects receiving active treatment and six other subjects receiving placebo. All drugs also met the prespecified criteria with this approach (Table 2; Fig. 3). An analysis of the 'by timepoint' effect on the PR and QRS intervals confirmed the known effects of quinine and dolasetron on cardiac conduction, with a largest mean $\Delta \Delta \mathrm{PR}$ effect $\sim 16 \mathrm{~ms}$ for both on day 2 and the largest mean $\Delta \Delta \mathrm{QRS}$ of 7.7 and $5.2 \mathrm{~ms}$ on day 2 , respectively.

\section{Implications of the IQ-CSRC Prospective Study}

The results from the IQ-CSRC study have been presented to the ICH E14 and S7B discussion group and were recently (December 2014) discussed at a public meeting at the FDA's White Oak campus, co-organized by CSRC/IQ/ FDA, with participation from main regulatory regions (USA/EU/Japan). The discussions were centered on the general applicability of the results, the lack of a positive control in studies intended to replace the TQT study, and limitations of the approach of applying ER analysis to routine $\mathrm{SAD} / \mathrm{MAD}$ studies. We believe that the results of the IQ-CSRC study provide clear support for replacing the TQT study with ECG assessment in routine clinical pharmacology studies, and would like to share some thoughts on topics that must be addressed for wider acceptance of this approach.

\subsection{Lack of Positive Control}

It is unrealistic to expect that early phase clinical studies will routinely include a pharmacological positive control; it is therefore important to consider how the study's sensitivity to detect small QT changes can be evaluated if data are to be used as a substitute for the TQT study. The positive control in a TQT study serves the purpose of demonstrating that the experimental conditions and the ECG methodology of the study are sensitive enough to detect a small effect of the investigational compound, should there be one. The positive control thereby provides reassurance against false negatives, i.e., the scenario where a study fails to detect a drug-induced QT effect, only to find out later in development (or after approval) that the drug causes proarrhythmias associated with pronounced QT prolongation. From a safety perspective, the risk of false negatives is therefore of key importance. However, this risk, appears small when ER analysis is applied to 
Table 1 Exposure response (QTc) analysis: the slope of the concentration/QTc relationship and the predicted $\Delta \Delta \mathrm{QTc}$ effect at peak plasma drug concentration

\begin{tabular}{|c|c|c|c|c|c|c|c|}
\hline \multirow[t]{2}{*}{ Drug } & \multirow{2}{*}{$\begin{array}{l}\text { Slope } \\
\text { [mean }(\mathrm{ms} \text { per } \mathrm{ng} / \mathrm{ml})]\end{array}$} & \multicolumn{2}{|l|}{$90 \% \mathrm{CI}$} & \multirow{2}{*}{$\begin{array}{l}C_{\max } \text { Day } 1 \\
{\left[\text { mean }(\mathrm{ng} / \mathrm{mL})^{\mathrm{a}}\right]}\end{array}$} & \multirow{2}{*}{$\begin{array}{l}\text { Predicted } \Delta \Delta \mathrm{QTc} \\
\text { effect mean }(\mathrm{ms})\end{array}$} & \multicolumn{2}{|c|}{$90 \% \mathrm{CI}$} \\
\hline & & LB & UB & & & LB & UB \\
\hline \multicolumn{8}{|l|}{ Positive drugs } \\
\hline Ondansetron & 0.033 & 0.025 & 0.042 & 284 & 9.7 & 6.2 & 12.8 \\
\hline Quinine & 0.004 & 0.0034 & 0.0047 & 3623 & 11.6 & 6.8 & 17.1 \\
\hline Hydrodolasetron & 0.021 & 0.013 & 0.028 & 211 & 7.4 & 3.0 & 11.0 \\
\hline Moxifloxacin & 0.0065 & 0.0059 & 0.0072 & 1862 & 14.5 & 10.5 & 17.7 \\
\hline Dofetilide $^{\mathrm{b}}$ & 22.2 & 18.9 & 25.6 & 0.42 & 10.5 & 6.3 & 14.9 \\
\hline \multicolumn{8}{|l|}{ Negative drug } \\
\hline Levocetirizine & 0.0014 & -0.0013 & 0.0041 & $1005^{\mathrm{a}}$ & 2.1 & -2.3 & 6.1 \\
\hline
\end{tabular}

Bold numbers indicate a statistically significant slope (i.e. the $L B$ of the $\mathrm{CI}$ is above $0 \mathrm{~ms}$ ) and that the predicted effect is above $10 \mathrm{~ms}$ (right hand column) for the positive drugs and below $10 \mathrm{~ms}$ for levocetirizine

The $90 \% \mathrm{CI}$ for the predicted QT effect was calculated using a bias-corrected non-parametric bootstrap procedure, which includes variability of $C_{\text {max }}$ $C I$ confidence interval, $C_{\max }$ geometric mean peak plasma level, $E_{\max }$ maximum response, $L B$ lower bound, $U B$ upper bound

${ }^{\text {a }} C_{\max }$ on day 2 for levocetirizine, $\Delta \Delta \mathrm{QTcF}$ placebo-adjusted change from baseline QTcF

${ }^{\mathrm{b}}$ For comparative purposes, parameters and predictions for dofetilide derived from a linear model are shown. Using an $E_{\max }$ ER model, the predicted mean effect on $\Delta \Delta \mathrm{QTcF}$ at $C_{\max }(0.42 \mathrm{ng} / \mathrm{mL})$ was similar: $11.6 \mathrm{~ms}(90 \% \mathrm{CI} 7.0-16.0)$

early phase QT studies, provided a wide range of plasma concentrations of the drug has been achieved and an intense ECG/pharmacokinetic schedule has been implemented using the same experimental conditions and ECG methodologies as in TQT studies. Since published examples are relatively few $[8,9,23,37,38]$ and not based on prospective series, a recently published report using a simulation approach can help to gain further understanding of the rate of false negatives and false positives in smallsized studies. A large number of small studies with 6-18 subjects receiving active treatment and six receiving placebo was simulated with data from five TQT studies; three studies with moxifloxacin with mean peak $\Delta \Delta \mathrm{QTcF}$ effect of $12.5,14.0$, and $8.0 \mathrm{~ms}$, one study with ketoconazole with a smaller QT effect ( $\Delta \Delta \mathrm{QTcF} 7.6 \mathrm{~ms})$, and one TQT study with a drug with a larger effect $(\Delta \Delta \mathrm{QTcF} 26 \mathrm{~ms})$. A total of 1000 studies were simulated for each of five sample sizes of subjects on active treatment $(n=6,9,12,15$, and 18) for each study, i.e., a total of 25,000 studies [39]. The criterion for negative QT assessment was based on ER analysis and was the same as in this study, i.e., a QT effect $(\Delta \Delta \mathrm{QTcF})$ exceeding $10 \mathrm{~ms}$ should be excluded. The rate of false negatives with a sample size of nine or more subjects receiving drug and six receiving placebo was $1 \%$ in two of the three moxifloxacin datasets and around $5 \%$ in the third. For ketoconazole, with a smaller peak effect than moxifloxacin, the rate of false negatives was larger with such small sample sizes (around 25-30 \%). Similar results, i.e., a rate of false negatives around $5 \%$, have been obtained with simulation studies performed on TQT study data submitted to the FDA (personal communication, Dr. Jiang Liu, scientific lead of the IRT). These simulations lend support to the claim that the risk of false-negative results is low when ER analysis is applied to data from small studies with drugs that have a threshold QT effect but call for confirmation from real-life studies. The rate of false positives is obviously also of interest, since a high rate would be ineffective from a resource perspective: this rate was below $20 \%$ with nine subjects receiving active (six receiving placebo) and near or below $10 \%$ with 12 subjects in the simulation study discussed above [39]. Which rate of false positives is acceptable will most likely vary among drug developers, since in these cases additional studies would be needed. In case of a small or ambiguous QT effect, a TQT may be the best option; whereas in case of a clear QT effect, further characterization of the effect in the targeted patient population may be needed.

In summary, the risk of false negatives with 'early QT assessment' seems acceptably low, in our view, provided the experimental conditions are similar to those in TQT studies and an intense ECG schedule with a high-quality technique has been implemented, paired with pharmacokinetic determination at each timepoint. It should also be emphasized that the confidence in a negative QT assessment in the absence of a positive control will be higher if plasma levels of the drug substantially exceeding therapeutic levels are achieved; if this is not the case, a positive control may be required to gain confidence in the negative results of the drug.

\subsection{How Generalizable are the Results: How to Ensure High Quality?}

Since a QT effect at the level of concern, i.e., around 10 $\mathrm{ms}$, could be detected in a consistent way in the IQ-CSRC 


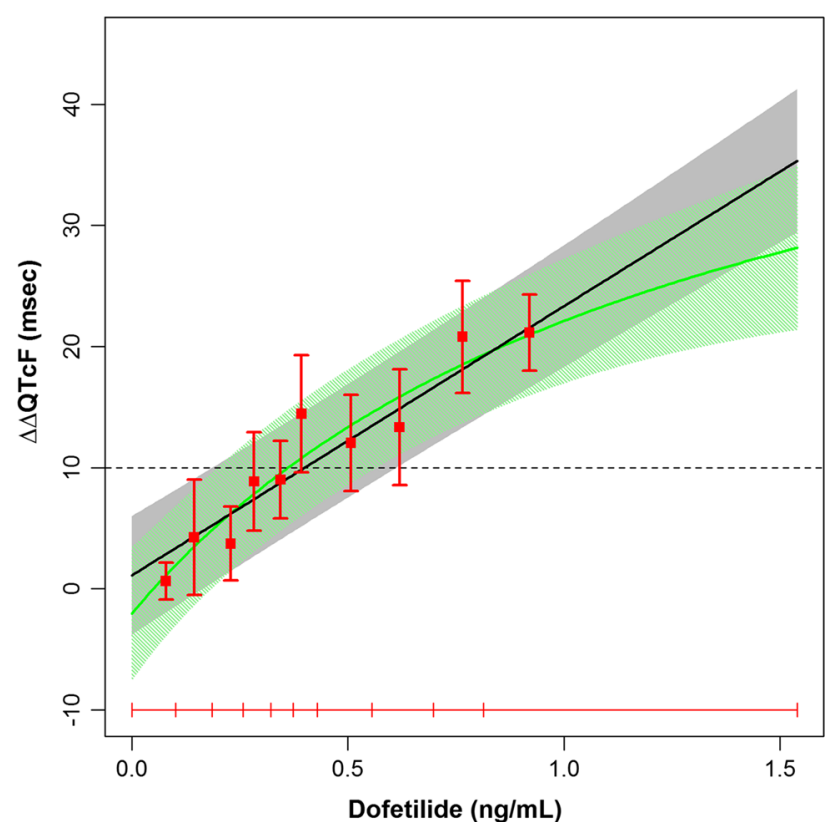

Fig. 1 The predicted effect of dofetilide on $\Delta \Delta \mathrm{QTcF}$ with a linear and a maximum response ER model (nine subjects receiving active drug and six receiving placebo for both study days). The solid black line with gray shaded area denotes the model-predicted mean placebo-adjusted $\Delta \mathrm{QTcF}$ with $90 \%$ confidence interval with the linear model, whereas the solid green line with the green shaded area shows the prediction with a maximum response model. The horizontal red line shows the range of plasma concentrations divided into deciles. Red squares with vertical bars denote the observed arithmetic means and $90 \%$ confidence intervals for the placebo-adjusted $\Delta \mathrm{QTcF}$ within each plasma-concentration decile. The placebo-adjusted $\Delta \mathrm{QTcF}$ was derived from the individual $\Delta \mathrm{QTcF}$ for the active subtracted by the mean predicted $\Delta \mathrm{QTcF}$ for placebo from the model. With both models, the slope of the exposure-response relation was statistically significant and the upper bound of the $90 \%$ confidence interval of the predicted QT effect at the observed peak plasma drug concentration on day $1(0.42 \mathrm{ng} / \mathrm{ml})$ was above $10 \mathrm{~ms}$

study, both in the primary analysis and in the sensitivity analyses, it may be claimed that the results provide validation of the approach of applying ER analysis to QT data obtained in small-sized studies to replace the TQT study; it can thus be argued that the results create confidence in the approach in general. The important question then becomes to what extent the results can be repeated using other clinical sites, experimental conditions, and ECG methodologies. For TQT studies, the positive control serves as the 'quality control' with certain preset criteria that are to be met. There is no reason to believe that the same level of high-quality data cannot be generated from SAD/MAD studies performed in healthy volunteers at experienced clinical sites, but some data-driven metrics of quality may be needed, at the least for the near- to mid-term future, as more experience is gained. Some research in this area has been performed using internal FDA data [40, 41], but tests have to be tailored and further defined to allow application to a typical SAD study without a full baseline day and small number of subjects. Quality tests could include metrics of heart rate stability within timepoints, a reproducible QT/RR curvature, within- and between-subject variability of the QT interval and the time course of the adaptation of the QT interval to changes in heart rate. While heart rate and QT variability can be evaluated from extracted ECGs at prespecified timepoints, other metrics may be based on a richer sample of QT/RR data from continuous ECG (Holter) recordings. This should be realistically achievable, given that most ECG studies in healthy subjects are currently performed using continuous recordings. Work has been initiated in this regard on the IQCSRC study dataset and will need to be tested on routine SAD/MAD studies to allow definition of useful metrics. Based on experience from TQT studies, it is also worth pointing out that stringent control of experimental conditions and the use of standardized ECG techniques will result in lower variability of the QT interval measurements. The objective of these studies is to exclude a small QT effect by using a non-inferiority approach (see criterion below) and consequently, the smaller the variability, the greater the chance is to exclude an effect. In a way, this serves as an internal quality control.

The underlying concept of ER analysis applied to early phase clinical data is that there should be no need to change the design and sample size of a typical SAD or MAD study; often plasma levels of the drug well above therapeutic levels are achieved and the total number of subjects across dose groups substantially exceeds the sample size $(n=9)$ of the IQ-CSRC study. Within this framework, certain prerequisites have to be met to support a request for a TQT waiver based on early QT assessment. Sufficiently high plasma concentrations of the parent and abundant metabolites are critically important to support a claim of the absence of a QT effect at clinically relevant concentrations; if justifiable from a tolerability perspective, achieved levels should substantially exceed the highest observed levels in patients. For drugs or metabolites with pronounced accumulation on multiple dosing, sufficiently high plasma levels may not be possible to achieve with a SAD study, and the ECG assessment would in such cases be better performed in a multiple-dose setting; these are the same type of considerations as those in the choice between a single-dose and multiple-dose TQT study.

ER analysis is mentioned in the ICH E14 'questions and answers' (Q\&A) document from March 2014 as 'promising in terms of enhancing our confidence to characterise QTc prolongation' [42]. Based on the extensive experience with ER analysis for evaluation of QT effects and the results of the IQ-CSRC study, it now seems timely to consider an expanded role for ER analysis in the definitive assessment of a drug's QT effect and whether ER analysis applied to 
Table 2 Exposure response (QTc) analysis: sensitivity analyses

\begin{tabular}{|c|c|c|c|c|c|c|c|}
\hline \multirow[t]{2}{*}{ Drug } & \multirow{2}{*}{$\begin{array}{l}\text { Slope } \\
{[\text { mean }(\mathrm{ms} \text { per } \mathrm{ng} / \mathrm{ml})]}\end{array}$} & \multicolumn{2}{|l|}{$90 \% \mathrm{CI}$} & \multirow{2}{*}{$\begin{array}{l}C_{\max } \\
\text { Day } 1(\mathrm{ng} / \mathrm{mL})^{\mathrm{a}}\end{array}$} & \multirow{2}{*}{$\begin{array}{l}\text { Predicted } \Delta \Delta \mathrm{QTc} \\
\text { effect }[\text { mean }(\mathrm{ms})]\end{array}$} & \multicolumn{2}{|c|}{$90 \% \mathrm{CI}$} \\
\hline & & LB & UB & & & LB & UB \\
\hline \multicolumn{8}{|l|}{ Ondansetron } \\
\hline Day 1 only & 0.032 & 0.022 & 0.043 & 284 & 9.5 & 7.2 & 13.5 \\
\hline Parallel design $\left(n=7^{\mathrm{c}}\right)$ & 0.042 & 0.031 & 0.052 & 259 & 10.2 & 6.8 & 13.5 \\
\hline \multicolumn{8}{|l|}{ Quinine } \\
\hline Day 1 only & 0.004 & 0.0031 & 0.0051 & 3623 & 9.8 & 6.7 & 17.3 \\
\hline Parallel design $\left(n=7^{\mathrm{c}}\right)$ & 0.0034 & 0.0027 & 0.0041 & 3643 & 9.5 & 4.8 & 14.5 \\
\hline \multicolumn{8}{|l|}{ Hydrodolasetron } \\
\hline Day 1 only & 0.016 & 0.0008 & 0.032 & 211 & 6.8 & 3.4 & 11.6 \\
\hline Parallel design $\left(n=7^{\mathrm{c}}\right)$ & 0.020 & 0.012 & 0.029 & 205 & 7.3 & 2.7 & 11.5 \\
\hline \multicolumn{8}{|l|}{ Moxifloxacin } \\
\hline Day 1 only & 0.0045 & 0.0025 & 0.0065 & 1862 & 11.7 & 10.6 & 17.9 \\
\hline Parallel design $\left(n=7^{\mathrm{c}}\right)$ & 0.0065 & 0.0058 & 0.0072 & 1708 & 13.3 & 9.6 & 17.0 \\
\hline \multicolumn{8}{|l|}{ Dofetilide $^{\mathrm{b}}$} \\
\hline Day 1 only & 28.7 & 20.6 & 36.7 & 0.42 & 11.3 & 6.1 & 14.6 \\
\hline Parallel design $\left(n=7^{\mathrm{c}}\right)$ & 25.0 & 20.9 & 29.0 & 0.40 & 8.9 & 5.1 & 13.9 \\
\hline \multicolumn{8}{|l|}{ Levocetirizine } \\
\hline Day 2 only & 0.00042 & -0.0032 & 0.0041 & $1005^{\mathrm{a}}$ & 2.0 & -2.6 & 6.0 \\
\hline Parallel design $\left(n=6^{\mathrm{c}}\right)$ & -0.0015 & -0.0046 & 0.0017 & 1014 & 0.3 & -4.7 & 4.2 \\
\hline
\end{tabular}

Bold numbers indicate a statistically significant slope (i.e. the $L B$ of the $\mathrm{CI}$ is above $0 \mathrm{~ms}$ ) and that the predicted effect is above $10 \mathrm{~ms}$ (right hand column) for the positive drugs and below $10 \mathrm{~ms}$ for levocetirizine

$C I$ confidence interval, $C_{\max }$ geometric mean peak plasma level, $E_{\max }$ maximum response, $L B$ lower bound, $U B$ upper bound

${ }^{\text {a }} C_{\max }$ on day 2 for levocetirizine

${ }^{\mathrm{b}}$ For comparative purposes, parameters and predictions for dofetilide derived from a linear model are shown

${ }^{c}$ For each drug, subjects also dosed with placebo were excluded in this post hoc analysis

early clinical phase data can serve as an alternative to the TQT study. For this purpose, the following criterion, analogous to the threshold in the TQT study, has been proposed [7] as a basis for a 'negative QT assessment', i.e., to demonstrate that a drug does not cause QT prolongation of concern:

- The upper bound of the two-sided $90 \% \mathrm{CI}$ of the predicted placebo-adjusted $\Delta \mathrm{QTc}$ should be below $10 \mathrm{~ms}$ at the highest clinically relevant plasma concentrations of the drug.

Therapeutic plasma concentrations and full pharmacokinetic characteristics will obviously not be known at the time of an early phase clinical study, which means that a two-tiered process would be needed, with confirmation of the utility of the dataset once the drug exposure in patients is well characterized, substantially later in development. If it then can be shown that achieved plasma concentrations in early phase clinical studies substantially exceed those seen in patients receiving chronic dosing, in terms of both parent drug and metabolites, a negative QT assessment using ER analysis may serve as a replacement for the TQT study [43].
Based on the increasing experience and confidence in ER analysis of ECG data among regulators and sponsors, and as a consequence of the discussions triggered by the IQ-CSRC study, the ICH E14 discussion group met in June 2015 in Fukuoka [44] and decided to revise the E14 guideline (personal communication Drs. Stockbridge and Garnett, representing FDA and Dr. Keirns, Astellas, representing US PhRMA on the E14 discussion group). This will be handled as an amended Q\&A document addressing the role of ER analysis of early phase clinical data, requirements for replacing the TQT study using this approach, and with advice on various aspects of the analysis and quality control of the data.

\section{Conclusions}

Based on extensive experience from TQT studies and from ECG assessment in patient trials, ER analysis has emerged as an important tool to evaluate the propensity of drugs to cause QT prolongation. A high concordance between the largest observed QT effect in TQT studies and the predicted QT effect at correspondingly high 


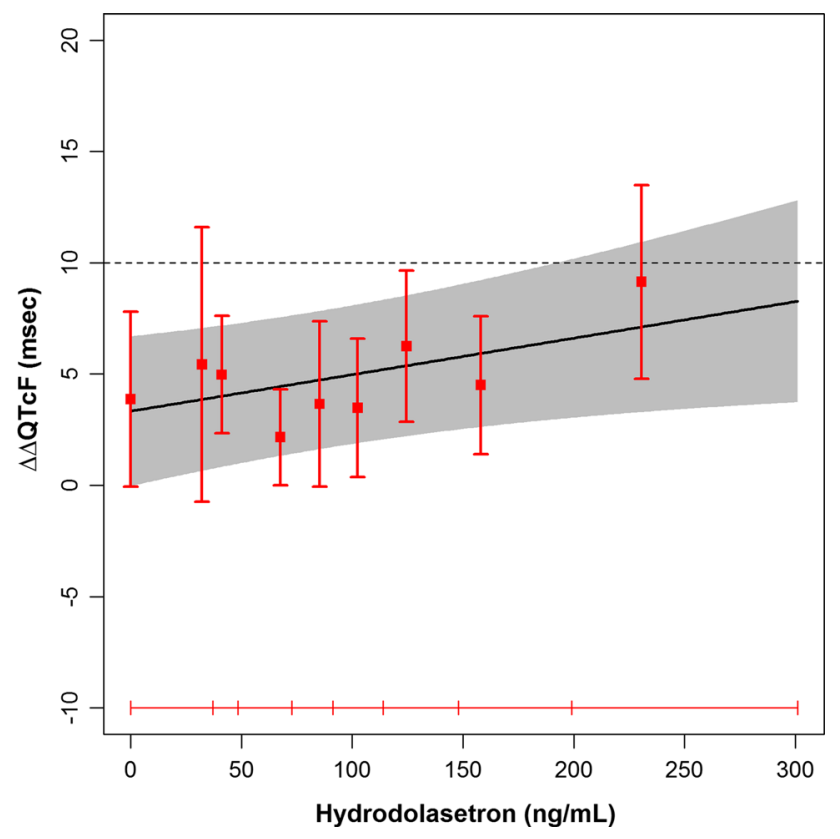

Fig. 2 The predicted effect of dolasetron (hydrodolasetron) on $\Delta \Delta \mathrm{QTcF}$ using data from the lower dose only (day 1) with nine subjects receiving active drug and six subjects receiving placebo; on this day, the largest mean $\Delta \Delta \mathrm{QTcF}$ across timepoints was only $6.5 \mathrm{~ms}$. The symbols are as in Fig. 1. The slope of the exposureresponse relation was statistically significant $(0.016 \mathrm{~ms}$ per $\mathrm{ng} / \mathrm{ml}$; $90 \%$ confidence interval 0.0008-0.032) and an effect on QTc above $10 \mathrm{~ms}$ could not be excluded (mean $6.8 \mathrm{~ms} ; 90 \%$ confidence interval 3.4-11.6) at the observed peak plasma drug concentration on day 1 $(211 \mathrm{ng} / \mathrm{ml})$

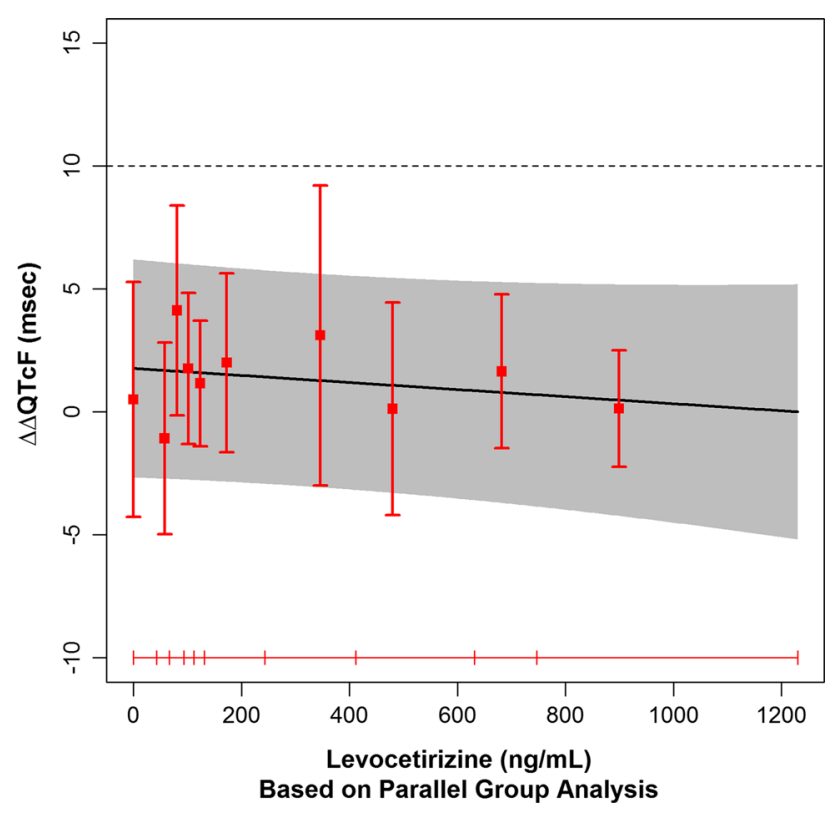

Fig. 3 Exposure-response analysis of the QT effect of levocetirizine using data from parallel groups of subjects with six subjects receiving active and six subject receiving placebo. The symbols are as in Fig. 1. An effect on $\Delta \Delta \mathrm{QTCF}$ exceeding $10 \mathrm{~ms}$ could be excluded throughout the observed concentration range plasma levels of the drug using ER analysis has been observed. The IQ-CSRC prospective study was designed to evaluate whether ER analysis applied to small-sized, early phase clinical studies can be used to detect drugs with a QT effect at the level of regulatory concern. The study correctly identified five 'QT-positive' compounds and excluded a QT effect with a 'QT-negative' drug, levocetirizine. The study thereby provided validation of the concept of using ER analysis applied to early phase clinical studies to provide definitive QT assessment and serve as a replacement for TQT studies. In consequence, the ICH E14 clinical guidance document will likely be revised to allow an expanded role for ER analysis.

\section{Compliance with Ethical Standards}

Funding The IQ-CSRC study discussed in this commentary was supported by the Consortium for Innovation and Quality in Pharmaceutical Development, the Cardiac Safety Research Consortium, and by the FDA. The study was sponsored and funded by iCardiac Technologies, Rochester, New York, USA. No sources of funding were used to assist in the preparation of this manuscript.

Conflicts of interest Borje Darpo holds stock and stock options in iCardiac Technologies. Jim Keirns is an employee of Astellas Pharma Global Development, Inc. Christine Garnett and Norman Stockbridge have no conflicts of interest that are directly relevant to the content of this study.

\section{References}

1. ICH Harmonized Tripartite Guideline E14. The clinical evaluation of QT/QTc interval prolongation and proarrhythmic potential for non-antiarrhythmic drugs. 2005. Available at: http://www.ich. org/fileadmin/Public_Web_Site/ICH_Products/Guidelines/Effic acy/E14/E14_Guideline.pdf. Accessed July 2015.

2. Shah RR. Drug-induced prolongation of the QT interval: why the regulatory concern? Fundam Clin Pharmacol. 2002;16(2): 119-24.

3. Stockbridge N, Morganroth J, Shah RR, Garnett C. Dealing with global safety issues: was the response to QT-liability of noncardiac drugs well coordinated? Drug Saf. 2013;36(3):167-82.

4. Malik M, Stockbridge N. Thorough QT studies and indirect causes of QTc changes. Pacing Clin Electrophysiol. 2012;35:1411-2.

5. Sager PT, Gintant G, Turner JR, Pettit S, Stockbridge N. Rechanneling the cardiac proarrhythmia safety paradigm: a meeting report from the Cardiac Safety Research Consortium. Am Heart J. 2014;167(3):292-300.

6. Bouvy JC, Koopmanschap MA, Shah RR, Schellekens H. The cost-effectiveness of drug regulation: the example of thorough QT/QTc studies. Clin Pharmacol Ther. 2012;91(2):281-8.

7. Darpo B, Garnett C. Early QT assessment: how can our confidence in the data be improved? $\mathrm{Br} \mathrm{J}$ Clin Pharmacol. 2013;76(5):642-8.

8. Rohatagi S, Carrothers TJ, Kuwabara-Wagg J, Khariton T. Is a thorough QTc study necessary? The role of modeling and simulation in evaluating the QTc prolongation potential of drugs. J Clin Pharmacol. 2009;49(11):1284-96.

9. Shah RR, Morganroth J. Early investigation of QTc liability: the role of multiple ascending dose (MAD) study. Drug Saf. 2012;35(9):695-709. 
10. Zhang J, Machado SG. Statistical issues including design and sample size calculation in thorough QT/QTc studies. J Biopharm Stat. 2008;18(3):451-67.

11. Garnett CE, Beasley N, Bhattaram VA, Jadhav PR, Madabushi R, Stockbridge N, et al. Concentration-QT relationships play a key role in the evaluation of proarrhythmic risk during regulatory review. J Clin Pharmacol. 2008;48(1):13-8.

12. Tornoe CW, Garnett CE, Wang Y, Florian J, Li M, Gobburu JV. Creation of a knowledge management system for QT analyses. J Clin Pharmacol. 2011;51(7):1035-42.

13. Asenapine (Saphris). QT-IRT consult to NDA 22,117. 2013. Available at: http://www.accessdata.fda.gov/drugsatfda_docs/ nda/2009/022117s000_MedR_P2.pdf. Accessed July 2015.

14. Citalopram (Celexa). FDA Drug Safety Communication: revised recommendations for Celexa (citalopram hydrobromide) related to a potential risk of abnormal heart rhythms with high doses. 2013. Available at: http://www.fda.gov/drugs/drugsafety/ ucm297391.htm. Accessed July 2015.

15. Dolasetron (Anzamet). US label. 2013. Available at: http://www. accessdata.fda.gov/drugsatfda_docs/label/2011/020623s009lbl. pdf. Accessed July 2015.

16. Florian J, Garnett CE, Nallani SC, Rappaport BA, Throckmorton DC. A modeling and simulation approach to characterize methadone QT prolongation using pooled data from five clinical trials in MMT patients. Clin Pharmacol Ther. 2012;91(4):666-72.

17. Ondansetron (Zofran). Drug Safety Communication-QT prolongation. 2012. Available at: http://www.fda.gov/Safety/ MedWatch/SafetyInformation/SafetyAlertsforHumanMedicalPro ducts/ucm310219.htm. Accessed July 2015.

18. Sertindole. Advisory Committee briefing material for NDA 20-644, Psychopharmacologic Drugs Advisory Committee March 27, 2009. Available at: http://www.fda.gov/downloads/advisory committees/committeesmeetingmaterials/drugs/psychopharmacolo gicdrugsadvisorycommittee/ucm161904.pdf. Accessed July 2015.

19. Vandetanib. (Caprelsa) Clinical Pharmacology review April 2011. 2013. Available at: http://www.accessdata.fda.gov/ drugsatfda_docs/nda/2011/022405Orig1s000ClinPharmR.pdf. Accessed July 2015.

20. Delisle BP, Anson BD, Rajamani S, January CT. Biology of cardiac arrhythmias: ion channel protein trafficking. Circ Res. 2004;94(11):1418-28.

21. Dennis A, Wang L, Wan X, Ficker E. hERG channel trafficking: novel targets in drug-induced long QT syndrome. Biochem Soc Trans. 2007;35(Pt 5):1060-3.

22. Dennis AT, Wang L, Wan H, Nassal D, Deschenes I, Ficker E. Molecular determinants of pentamidine-induced hERG trafficking inhibition. Mol Pharmacol. 2012;81(2):198-209.

23. Westerberg G, Chiesa JA, Andersen CA, Diamanti D, Magnoni L, Pollio G, et al. Safety, pharmacokinetics, pharmacogenomics and QT concentration-effect modelling of the SirT1 inhibitor selisistat in healthy volunteers. $\mathrm{Br} \mathrm{J}$ Clin Pharmacol. 2015;79(3):477-91.

24. International Consortium for Innovation and Quality in Pharmaceutical Development. http://www.iqconsortium.com/. Accessed July 2015.

25. Cardiac Safety Research Consortium. https://www.cardiac-safety. org/. Accessed July 2015.

26. Darpo B, Sarapa N, Garnett C, Benson C, Dota C, Ferber G, et al. The IQ-CSRC prospective clinical Phase 1 study: "Can early QT assessment using exposure response analysis replace the thorough QT study?". Ann Noninvasive Electrocardiol. 2014;19(1):70-81.

27. Darpo B, Benson C, Dota C, Ferber G, Garnett C, Green CL, et al. Results from the IQ-CSRC prospective study support replacement of the thorough QT study by QT assessment in the early clinical phase. Clin Pharmacol Ther. 2015;97(4):326-35.

28. Zofran (ondansetron hydrochloride). US label. Available at: http://www.accessdata.fda.gov/drugsatfda_docs/label/2012/0200 07s043lbl.pdf. Accessed July 2015.

29. Qualaquin (quinine sulphate). Clinical pharmacology and biopharmaceutics review(s). 2005. Available at: http://www. accessdata.fda.gov/drugsatfda_docs/nda/2005/021799s000_Clin PharmR.pdf. Accessed July 2015.

30. Qualaquin (quinine sulphate). US label. 2013. Available at: http:// www.accessdata.fda.gov/drugsatfda_docs/label/2013/021799s02 3lbl.pdf. Accessed July 2015.

31. Anzemet (dolasetron mesylate). US label. 2013. Available at: http://www.accessdata.fda.gov/drugsatfda_docs/label/2013/0206 23s010lbl.pdf,020624s023lbl.pdf. Accessed July 2015.

32. Avelox (moxifloxacin hydrochloride). US label. 2013. Available at: http://www.accessdata.fda.gov/drugsatfda_docs/label/2013/ 021085s057,021277s054lbl.pdf. Accessed July 2015.

33. Florian JA, Tornoe CW, Brundage R, Parekh A, Garnett CE. Population pharmacokinetic and concentration-QTc models for moxifloxacin: pooled analysis of 20 thorough QT studies. J Clin Pharmacol. 2011;51(8):1152-62.

34. Tikosyn (dofetilide). US label. 1999. Available at: http://www. accessdata.fda.gov/drugsatfda_docs/label/1999/20931lbl.pdf. Accessed July 2015.

35. Hulhoven R, Rosillon D, Letiexhe M, Meeus MA, Daoust A, Stockis A. Levocetirizine does not prolong the QT/QTc interval in healthy subjects: results from a thorough QT study. Eur J Clin Pharmacol. 2007;63(11):1011-7.

36. Xyzal (levocetirizine dihydrochloride). US label. 2012. Available at: http://www.accessdata.fda.gov/drugsatfda_docs/label/2012/ 022064s024,022157s008lbl.pdf. Accessed July 2015.

37. Malik M, Hnatkova K, Ford J, Madge D. Near-thorough QT study as part of a first-in-man study. J Clin Pharmacol. 2008;48(10):1146-57.

38. Sparve E, Quartino AL, Luttgen M, Tunblad K, Gardlund AT, Falting J, et al. Prediction and modeling of effects on the QTc interval for clinical safety margin assessment, based on singleascending-dose study data with AZD3839. J Pharmacol Exp Ther. 2014;350(2):469-78.

39. Ferber G, Zhou M, Darpo B. Detection of QTc effects in small studies-implications for replacing the thorough QT study. Ann Noninvasive Electrocardiol. 2014. doi:10.1111/anec.12227 (Epub ahead of print).

40. Johannesen L, Garnett C, Malik M. Electrocardiographic data quality in thorough QT/QTc studies. Drug Saf. 2014;37(3):191-7.

41. Malik M, Zhang J, Johannesen L, Hnatkova K, Garnett C. Assessing electrocardiographic data quality and possible replacement of pharmacologic positive control in thorough QT/ QTc studies by investigations of drug-free QTc stability. Heart Rhythm. 2011;8(11):1777-85.

42. ICH E14 Questions and answers. 2014. http://www.ich.org/ fileadmin/Public_Web_Site/ICH_Products/Guidelines/Efficacy/ E14/E14_QAs_R2_Step4.pdf. Accessed July 2015.

43. Nelson $\mathrm{CH}$, Fang L, Cheng F, Wang L, Hepner M, Lin J, et al. Concentration-QTc modeling in first-in-human study to assess the effect of the investigational drug GS-4997 on cardiac repolarization. Poster presented at ASCPT 2015.

44. ICH Steering Committee Agenda papers 6-11 June. 2015. http:// www.ich.org/fileadmin/Public_Web_Site/Meetings/SC_Meet ings/ICH30_43_electronic_SC_Agenda_Paper_Fukuoka_2015. pdf. Accessed July 2015. 